\title{
Contact Analysis of Mobility Devices Based on Tension
}

\author{
Satoshi Takayama ${ }^{1}$, Shoichiro Takehara ${ }^{2, *} \mathbb{D}$ and Ryota Yuasa ${ }^{1}$ \\ 1 Department of Science and Technology, Graduate School of Sophia University, 7-1 Kioi-cho, Chiyoda-ku, \\ Tokyo 102-8554, Japan; s-takayama-414@eagle.sophia.ac.jp (S.T.); r-yuasa-8gm@eagle.sophia.ac.jp (R.Y.) \\ 2 Department of Engineering and Applied Sciences, Sophia University, 7-1 Kioi-cho, Chiyoda-ku, \\ Tokyo 102-8554, Japan \\ * Correspondence: stakeha@sophia.ac.jp; Tel.: +81-3-3238-3863
}

\section{check for}

updates

Citation: Takayama, S.; Takehara, S.; Yuasa, R. Contact Analysis of Mobility Devices Based on Tension. Modelling 2021, 2, 370-384. https:// doi.org/10.3390/modelling2030020

Academic Editor: José A.F.O. Correia

Received: 19 July 2021

Accepted: 18 August 2021

Published: 20 August 2021

Publisher's Note: MDPI stays neutral with regard to jurisdictional claims in published maps and institutional affiliations.

Copyright: (C) 2021 by the authors. Licensee MDPI, Basel, Switzerland. This article is an open access article distributed under the terms and conditions of the Creative Commons Attribution (CC BY) license (https:// creativecommons.org/licenses/by/ $4.0 /)$.

\begin{abstract}
Advances in space technology have opened up opportunities for human beings to work in outer space. It is expected that the upsizing of manned space facilities, such as the International Space Station, will further this trend. A unique means of transportation is necessary to ensure that human beings can move about effectively in microgravity environments. Here, we propose a tether-based mobility system that moves the user by winding a tether attached to a structure at the destination. To overcome the attitude instability of the user during tether winding, the Tether Space Mobility Device (TSMD) attitude control method for winding a tether is applied and examined through numerical analysis. The proposed analytical model for motion analysis consists of one flexible body and three rigid bodies. The contact force between the tether and the TSMD inlet is determined. Using the numerical analysis model, we investigated the effect of slit shape during tether extension and winding.
\end{abstract}

Keywords: contact modeling; space robot; tether; interaction; ANCF

\section{Introduction}

Mechanical systems, such as elevators and cranes, utilize tension. In such systems, an object is moved by the tension acting on a wire. Because wires are lightweight and can be easily stored, large-scale systems can be constructed. Systems based on wires for special environments such as outer space and oceans have been proposed, including a tether satellite system [1], in which a main satellite and a small sub-satellite are connected by a tether (a robot can be connected to the tether for in-orbit servicing), and a tether space robot [2]. The present authors previously proposed the Tether Space Mobility Device (TSMD) [3], which is a mobile device that uses a tether for transportation under microgravity conditions. As shown in Figure 1, this system moves the target by ejecting the tip of the device, capturing it at the destination, and collecting the tether bound to the tip. In such a system, when the wire is extended and collected, it may interfere with the connected device during complicated movements. This problem, called the spaghetti problem [4], occurs when a large vibration is generated when a wire is moved into a slit in an elastic wall at a specific winding speed. The vibration depends on the shape of the slit of the take-up device. Slit design is thus important for systems that use wire extension and winding. Wire vibration can also affect the attitude of the entire system. However, it has not been sufficiently clarified how wire vibration is affected by the slit shape or when the slit in the elastic wall itself is accompanied by movement. To consider these problems, it is important to analyze the effect of slit shape with movement of the elastic wall on the movement of the entire system using numerical simulation.

In this paper, we model the inlet of the TSMD in detail and constructed a numerical analysis model considering the length change, large deformation, and large displacement of the tether. Then, using this numerical analysis model, we investigate the effect of slit shape during tether extension and winding. 


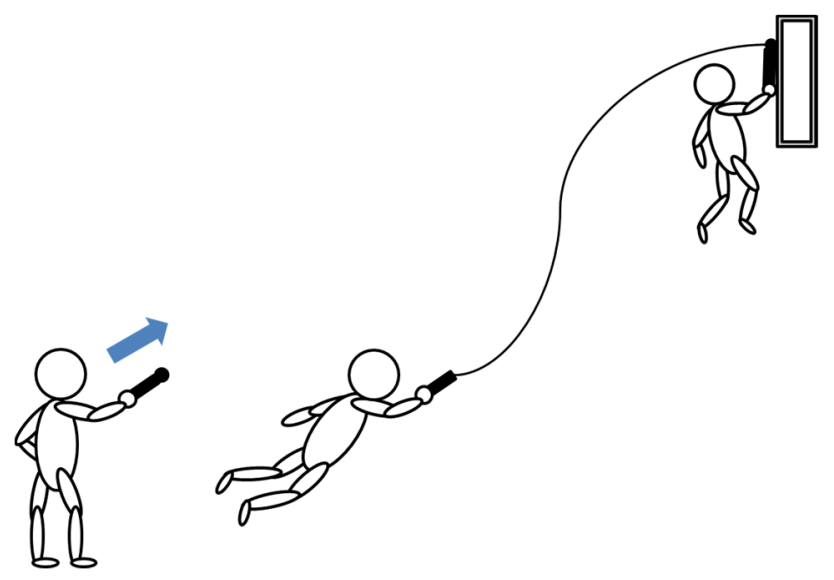

Figure 1. Overview of TSMD.

\section{Analysis Model}

Figure 2 shows the analytical model of the TSMD. This model, which comprises one flexible body and three rigid bodies, can be used to simulate a series of movements that involve tether extension and winding. In this model, the motion of the system is limited to a plane. The tether is modeled as a flexible body. The TSMD inlet (with the slit in the elastic wall), the TSMD body, and a human analog are modeled as rigid bodies. Although the inlet is located inside the TSMD, it is considered as an independent rigid body because an actuator may be mounted in the future. Here, a pin joint constraint is applied between rigid bodies 1 and 2 and between rigid bodies 2 and 3 . In addition, extending and winding the tether is expressed by applying a drive constraint between the node of the element at the rear end of the tether and rigid body 2 . To express the interaction between the tether and the rigid system, the tether is extended and its contact with rigid bodies 1 and 2 is simulated. Contact is judged at each node of the element of the tether. We then calculate the force.

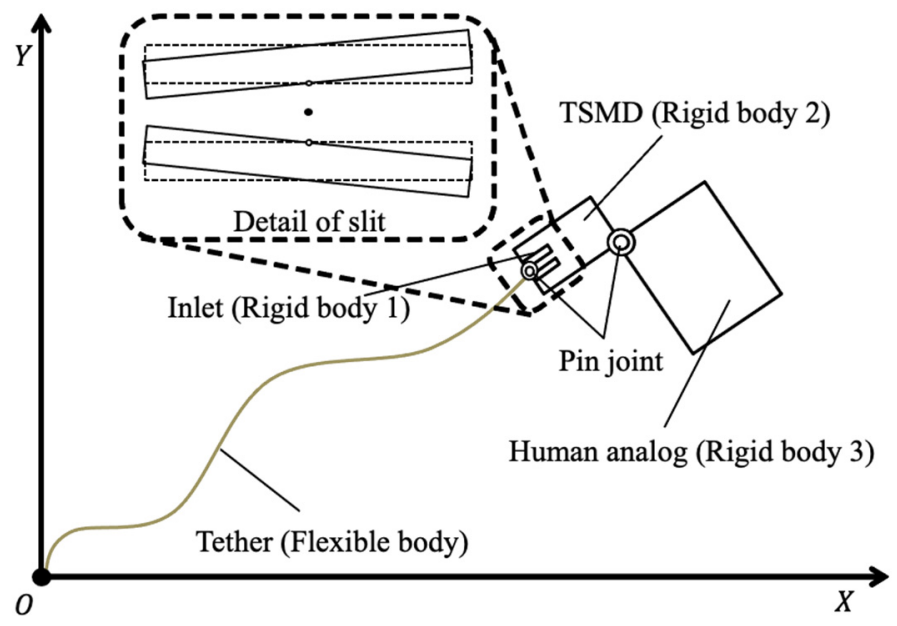

Figure 2. Outline of analytical model.

\subsection{Formulation of Analytical Model}

In the formulation of the tether, the absolute nodal coordinate formulation [5], proposed by Shabana et al., is applied to the flexible body to consider large deformation and large displacement. The model S_L1 [6], proposed by Wago et al., is used for the axial elastic force, and the model T1 [7], proposed by Berzeri and Shabana, is used for the bending elastic force. In the present paper, only an overview is shown about the formulation. The 
details of the formulation can be found elsewhere [8,9]. The motion equation of the flexible body finally becomes as follows:

$$
\mathbf{M}_{a} \ddot{\mathbf{e}}+\left(\mathbf{K}_{t}+\mathbf{K}_{l}\right) \mathbf{e}=\mathbf{Q}_{f}
$$

where $\mathbf{M}_{a}$ is the mass matrix, $\mathbf{e}$ is a node coordinate vector, $\mathbf{K}_{t}$ is flexural rigidity matrix, $\mathbf{K}_{l}$ is a nonlinear axial stiffness matrix and $\mathbf{Q}_{f}$ is the external force term including gravity, $\mathbf{M}_{a}$ is given by the following equation:

$$
\mathbf{M}_{a}=\int_{V} \rho \mathbf{S}^{T} \mathbf{S} d V
$$

where $\rho$ is the density of the flexible body, the shape function $\mathbf{S}$ is determined based on the assumption of a Bernoulli-Eulerian beam. $\mathbf{K}_{t}$ is expressed by following equation:

$$
\mathbf{K}_{t}=\int_{0}^{l_{e}} E_{t} I\left(\frac{d^{2} \mathbf{S}}{d x^{2}}\right)^{T}\left(\frac{d^{2} \mathbf{S}}{d x^{2}}\right) d x
$$

where $l_{e}$ is the length of the element of flexible body, and we assume that $x$ is the position of arbitrary point from element node before deformation. $E_{t}$ is the elastic modulus in the bending direction. $\mathbf{K}_{l}$ is expressed by following equation:

$$
\mathbf{K}_{l}=\bar{\varepsilon}_{S_{-} L 1} \int_{0}^{l_{e}} E_{l} A\left(\frac{d \mathbf{S}}{d x}\right)^{T}\left(\frac{d \mathbf{S}}{d x}\right) d x
$$

where $\bar{\varepsilon}_{S_{-} L 1}$ is the average axial strain of the element of flexible body, $E_{l}$ is the elastic modulus in the axial direction and $A$ is the cross-sectional area.

Next, we construct a differential algebraic equation using the expansion method [5]. The equation of motion of the system can be expressed as:

$$
\left[\begin{array}{cc}
\mathbf{M} & \boldsymbol{\Phi}_{\mathbf{q}}^{T} \\
\boldsymbol{\Phi}_{\mathbf{q}} & \mathbf{0}
\end{array}\right]\left[\begin{array}{c}
\ddot{\mathbf{q}} \\
\lambda
\end{array}\right]=\left[\begin{array}{c}
\mathbf{Q} \\
\gamma
\end{array}\right]
$$

where $\mathbf{M}=\operatorname{diag}\left(\mathbf{M}_{a}, \mathbf{M}_{r}\right)$ is the mass matrix, $\mathbf{q}=\left[\begin{array}{ll}\mathbf{e}_{a} & \mathbf{q}_{r}\end{array}\right]^{T}$ is the generalized coordinates, and $\mathbf{Q}=\left[\begin{array}{ll}\mathbf{Q}_{a} & \mathbf{Q}_{r}\end{array}\right]^{T}$ is the external force term. $\mathbf{M}_{a}$ is the mass matrix of the tether, $\mathbf{M}_{r}=\operatorname{diag}\left(m_{r 1}, m_{r 1}, I_{r 1}, m_{r 2}, m_{r 2}, I_{r 2}, m_{r 3}, m_{r 3}, I_{r 3}\right)$ is the mass matrix of the rigid system, $\mathbf{e}_{a}$ is the generalized coordinates of the flexible body tether, $\mathbf{q}_{r}$ is the generalized coordinates of the rigid system, $\mathbf{Q}_{a}$ is the generalized force vector of the tether, and $\mathbf{Q}_{r}$ is the generalized force vector of the rigid system. $\boldsymbol{\Phi}_{\mathrm{q}}$ is the Jacobean matrix, $\lambda$ are Lagrange multipliers, and $\gamma$ is the acceleration equation. This equation is obtained by differentiating the constraint twice with respect to time. Next, we show the system constraints. Pin joints connect rigid bodies 1 and 2, and rigid bodies 2 and 3 . Then, the system constraints are given by:

$$
\left[\begin{array}{l}
\mathbf{R}_{1}+\mathbf{T}_{1} \overline{\mathbf{u}}_{2}^{1}-\mathbf{R}_{2}-\mathbf{T}_{2} \overline{\mathbf{u}}_{1}^{2} \\
\mathbf{R}_{2}+\mathbf{T}_{2} \overline{\mathbf{u}}_{3}^{2}-\mathbf{R}_{3}-\mathbf{T}_{3} \overline{\mathbf{u}}_{2}^{3}
\end{array}\right]=\mathbf{0}
$$

where $\mathbf{R}_{j}$ is the position vector of the center of rigid body $j$ in absolute coordinates, $\mathbf{T}_{j}$ is transformation matrix from the local coordinate system to the absolute coordinate system, and $\overline{\mathbf{u}}_{i}^{i}$ is a position vector up to the joint point of rigid body $j$ in local coordinates. Then, the relative angle between the rigid bodies is determined by the driving constraints and is described as:

$$
\left[\begin{array}{c}
\theta_{r 1}-\theta_{r 2}-\theta_{r 12}(t) \\
\theta_{r 2}-\theta_{r 3}-\theta_{r 23}(t)
\end{array}\right]=\mathbf{0}
$$

where $\theta_{r j}$ are their rotation angles $(j=1,2,3), \theta_{r 12}(t)$ is the relative angle between rigid bodies 1 and 2 , and $\theta_{r 23}(t)$ is the relative angle between rigid bodies 2 and 3 . 
In the present paper, we do not consider the relative motion between the rigid bodies, and set the relative angles between rigid bodies as $\theta_{r 12}(t)=\theta_{r 23}(t)=0$. Extension and winding of the tether is expressed by displacing the node at the end of the tether at velocity $V$ in the $X$ direction in the local coordinate system of rigid body 2 . Therefore, if the total length of the tether is $l_{0}$, the node constraint at the end of the tether and rigid body 2 can be described as:

$$
\left[\begin{array}{c}
e_{5}^{(n)} \\
e_{6}{ }^{(n)}
\end{array}\right]-\mathbf{R}_{2}-\mathbf{T}_{2}\left(\overline{\mathbf{u}}_{1}^{2}+\left[\begin{array}{c}
\int_{0}^{t} V d t+l_{0} \\
0
\end{array}\right]\right)=\mathbf{0}
$$

where $e_{5}^{(n)}, e_{6}{ }^{(n)}$ represent the node of the element at the rear end of the tether. Therefore, the constraint equations for the system $\boldsymbol{\Phi}$ can be written as:

$$
\boldsymbol{\Phi}=\left[\begin{array}{c}
{\left[\begin{array}{c}
e_{5}{ }^{(n)} \\
e_{6}(n)
\end{array}\right]-\mathbf{R}_{2}-\mathbf{T}_{2}\left(\overline{\mathbf{u}}_{1}^{2}+\left[\begin{array}{c}
\int_{0}^{t} V d t+l_{0} \\
0
\end{array}\right]\right)} \\
\mathbf{R}_{1}+\mathbf{T}_{1} \overline{\mathbf{u}}_{2}^{1}-\mathbf{R}_{2}-\mathbf{T}_{2} \overline{\mathbf{u}}_{1}^{2} \\
\mathbf{R}_{2}+\mathbf{T}_{2} \overline{\mathbf{u}}_{3}^{2}-\mathbf{R}_{3}-\mathbf{T}_{3} \overline{\mathbf{u}}_{2}^{3} \\
\theta_{r 1}-\theta_{r 2}-\theta_{r 12}(t) \\
\theta_{r 2}-\theta_{r 3}-\theta_{r 23}(t)
\end{array}\right]=\mathbf{0}
$$

\subsection{Contact Force between Flexible and Rigid Bodies}

Here, we derive the formulation of the contact force exerted by the rigid system on the tether. In this model, the modeling method for the spaghetti problem [10] proposed by Kobayashi et al. is applied to the absolute nodal coordinate formulation. The contact force exerted by the elastic wall inside the rigid body on the tether is defined by a spring element and a damper element. Figure 3 shows elements of flexible body sucked into rigid body 1. The number of the node located outside of and closest to the tip of rigid body 1 is set to $i$, whereas the number of the node located inside of and closest to the tip of rigid body 1 is set to $i+1$. $\left(\bar{x}_{i+1}^{1}, \bar{y}_{i+1}^{1}\right)$ is the position vector of $i+1$ th node in the local coordinate system for the rigid body 1 . To express the suction at the tip of the inlet, the contact force is divided into force $\mathbf{F}_{1}$ (exerted by the inner wall of the rigid body on the node of the beam element completely sucked into the rigid body) and force $\mathbf{F}_{2}$ (exerted on the beam element being moved from the tip of the suction port of the rigid body).

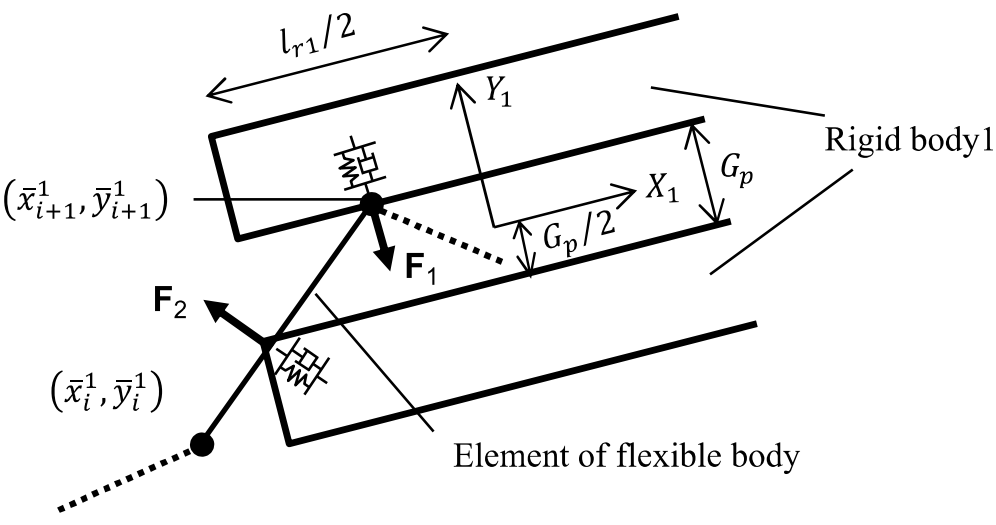

Figure 3. Element of flexible body sucked into rigid body 1. 
The force $\mathbf{F}_{1}$ from the inner wall of rigid body 1 on the $i+1$ th node can be expressed in the absolute coordinate system as follows:

$$
\begin{array}{ll}
\mathbf{F}_{1}=\mathbf{T}_{1}\left[\begin{array}{cc}
-\mu_{1}\left[-k_{1}\left\{\bar{y}_{i+1}^{1}-\operatorname{sgn}\left(\bar{y}_{i+1}^{1}\right) \frac{G_{p}}{2}\right\}-c_{1} \dot{\bar{y}}_{i+1}^{1}\right] \\
-k_{1}\left\{\bar{y}_{i+1}^{1}-\operatorname{sgn}\left(\bar{y}_{i+1}^{1}\right) \frac{G_{p}}{2}\right\}-c_{1} \dot{\bar{y}}_{i+1}^{1}
\end{array}\right] & \left(\left|\bar{y}_{i+1}^{1}\right| \geq \frac{G_{p}}{2}\right) \\
\mathbf{F}_{1}=\mathbf{0} & \left(\left|\bar{y}_{i+1}^{1}\right|<\frac{G_{p}}{2}\right)
\end{array}
$$

where $\mu_{1}$ is the friction coefficient of inner wall of inlet, $G_{p}$ is the slit width, $k_{1}$ and $c_{1}$ are the spring constant and the damping coefficient for the elastic wall in rigid body 1 , while taking contact rigidity into account. The force $\mathbf{F}_{2}$ from the tip of rigid body 1 on the $i+1$ th node can be expressed in the absolute coordinate system as follows:

$$
\mathbf{F}_{2}=\mathbf{T}_{1}\left(\overline{\mathbf{F}}_{k 2}^{1}+\overline{\mathbf{F}}_{f 2}^{1}\right)
$$

where $\overline{\mathbf{F}}_{k 2}^{1}$ is the contact force of the edge of rigid body 1 in the local coordinate system. $\overline{\mathbf{F}}_{k 2}^{1}$ can be expressed as follows:

$$
\overline{\mathbf{F}}_{k 2}^{1}=\left[\begin{array}{c}
\bar{F}_{k 2 x}^{1} \\
\bar{F}_{k 2 y}^{1}
\end{array}\right]=-k_{2} \Delta \overline{\mathbf{r}}_{2}^{1}-c_{2} \Delta \dot{\overline{\mathbf{r}}}_{2}^{1}
$$

where $k_{2}$ and $c_{2}$ are the spring constant and the damping coefficient, respectively, for the elastic wall in the vicinity of the tip of rigid body $1, \Delta \overrightarrow{\mathbf{r}}_{2}^{1}$ is the deformation of the local coordinate system of rigid body $1 . \overline{\mathbf{F}}_{f 2}^{1}$ is the frictional force in the local coordinate system from the tip of rigid body 1 on the beam element. $\overline{\mathbf{F}}_{f 2}^{1}$ is described as:

$$
\overline{\mathbf{F}}_{f 2}^{1}=\mu_{2}\left[\begin{array}{c}
-\operatorname{sgn}\left(\dot{\bar{x}}_{i}^{1}\right)\left|\bar{F}_{k 2 y}^{1}\right| \\
-\operatorname{sgn}\left(\dot{\bar{y}}_{i}^{1}\right)\left|\bar{F}_{k 2 x}^{1}\right|
\end{array}\right]
$$

where $\mu_{2}$ is the friction coefficient between the beam element and the tip of rigid body 1 .

\subsection{Adjustment of Slit Shape}

As an initial study on slit shape, we consider the case where the inclination of the elastic wall inside the inlet is changed. Figure 4 shows the inlet after the shape change. When the elastic wall is tilted, it rotates around the center position of the inlet on the elastic wall (white circle in Figure 4). For calculating the contact force, the inlet is divided into upper and lower sides. The calculation is performed in each coordinate system. Here, the upper inlet is taken as an example. Figure 5 shows the upper inlet tilted by angle $\gamma$. Hereafter, the inlet after the shape change is denoted as rigid body $1^{\prime}$. If the position vector of rigid body $1^{\prime}$ in the local coordinate system of rigid body 1 is $\left(\bar{x}_{i+1}^{1^{\prime}}, \bar{y}_{i+1}^{1^{\prime}}\right)$, we can obtain the following equation:

$$
\left[\begin{array}{l}
\bar{x}_{i+1}^{\prime} \\
\bar{y}_{i+1}^{\prime}
\end{array}\right]=\mathbf{T}_{1}^{\prime}\left(\left[\begin{array}{l}
\bar{x}_{i+1}^{1} \\
\bar{y}_{i+1}^{1}
\end{array}\right]-{ }^{-1} \mathbf{u}_{1^{\prime}}\right)
$$

where $\mathbf{T}_{1}^{\prime}$ is a coordinate transformation matrix that rotates the elastic wall angle $\gamma$ from the local coordinate system $O^{1}-X^{1} Y^{1}$ of rigid body $1 . \overline{\mathbf{u}}_{1^{\prime}}^{1}$ is a position vector up to the position of the center of gravity of rigid body $1^{\prime}$ in the local coordinate system of rigid body 1 , and is expressed by the following equation:

$$
\overline{\mathbf{u}}_{1^{\prime}}^{1}=\frac{G_{p}}{2}\left[\begin{array}{c}
\sin \gamma \\
1-\cos \gamma
\end{array}\right]
$$


Using the same method for the lower inlet, the contact force when the shape of the inlet is changed can be obtained.

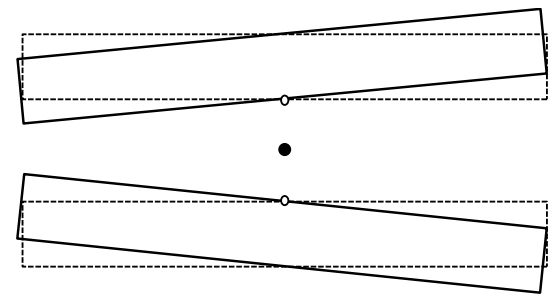

Figure 4. Outline of deformed inlet.

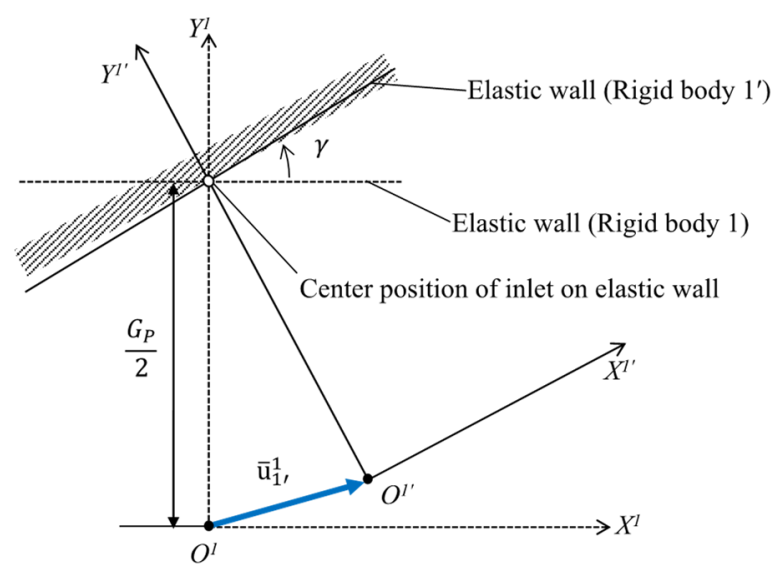

Figure 5. Outline of deformed upper inlet.

\subsection{Posture Control Method}

We apply winding control that focuses on the change in the kinetic energy of the rigid system and converges the angular velocity of the rigid system generated during tether winding to $0 \mathrm{deg} / \mathrm{s}$ [11]. Figure 6 shows a model of the TSMD with the tether stretched. Here, let $r$ be the target length of the uncollected tether, defined as:

$$
\left\{\begin{array}{ll}
r=d+u_{+} & \dot{\theta} \sin (\varphi-\theta+\alpha) \geq 0 \\
r=d+u_{-} & \dot{\theta} \sin (\varphi-\theta+\alpha)<0
\end{array}, u_{+} \geq 0, u_{-} \leq 0\right.
$$

where $d$ is the distance from the origin of the the absolute coordinate system to the tip of the inlet, $\varphi$ is the angle between the $X$ axis of the absolute coordinate system and the tether, $\theta$ is the rotation angle of the rigid system, and $\alpha$ is the angle of the tip position of the inlet in the local coordinate system. In addition, $u_{+}$is the control input when $\dot{\theta} \sin (\varphi-\theta+\alpha) \geq 0$, and $u_{-}$is the control input when $\dot{\theta} \sin (\varphi-\theta+\alpha)<0$. This value can be set arbitrarily to obtain the control effect. With the above definition of $r$ as Equation (16), the tether deflects when the tension is working to increase the kinetic energy of the rigid body, and the tether stretches when the tension works to reduce the kinetic energy of the rigid body. The angular velocity of the rigid system eventually converges to zero deg/s. 


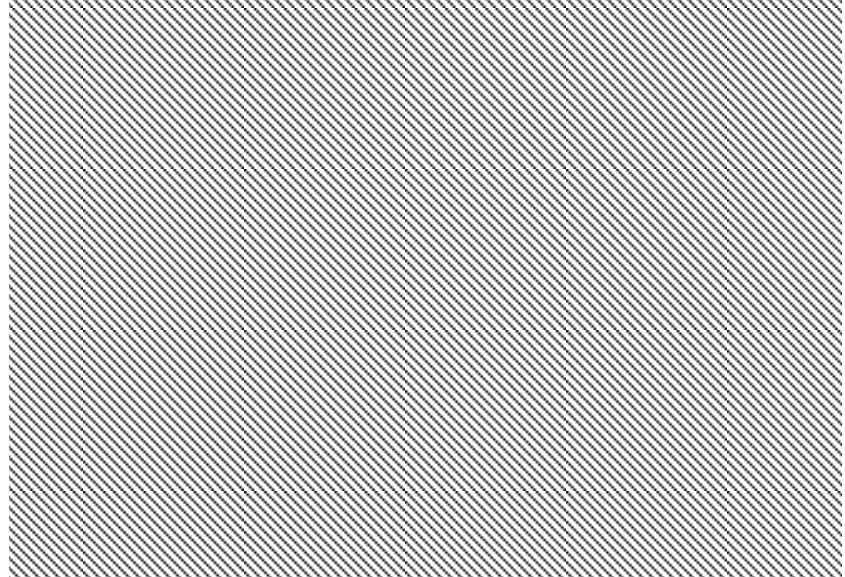

Figure 6. Model of TSMD when tether is under tension.

\section{Evaluation of Posture Control Method for Each Shape}

We now compare different slit shapes in the elastic wall. The attitude control method is evaluated based on the numerical simulation results.

\subsection{Analysis Conditions}

\subsubsection{Elastic Wall Slit Shape}

Figure 7 shows an outline of the slit shape as a longitudinal cross-sectional view of the inlet. Figure 8 shows the three types of slit shape examined here. In these figures, it is assumed that the tether is collected from the left side to the right side. Diameter $d_{1}$ at the tip of the inlet and diameter $d_{2}$ at the rear end of the inlet are used as parameters. For the three types of shape, the slit width is uniform, the inlet tip narrows, and the inlet tip widens, respectively. Table 1 shows the $d_{1}$ and $d_{2}$ values for each condition. The smallest diameter is $1 \mathrm{~mm}$, which is the diameter of the tether used in the "STARS Project" ultra-small tether extension satellite demonstration experiment in space [12]. Considering that the inlet is mounted inside the TSMD, the largest diameter is $9 \mathrm{~mm}$.

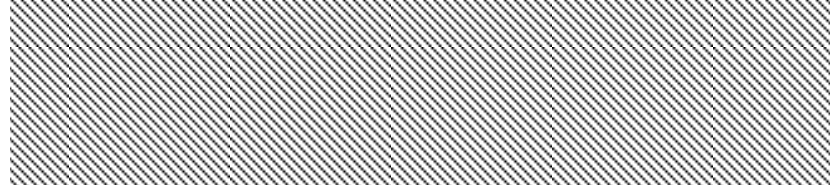

Figure 7. Longitudinal cross-sectional view of TSMD inlet.

Table 1. Parameters for various simulation conditions.

\begin{tabular}{ccc}
\hline Condition & $d_{1}(\mathbf{m m})$ & $d_{2}(\mathbf{m m})$ \\
\hline $1-1$ & 1 & 1 \\
$1-5$ & 1 & 5 \\
$1-9$ & 1 & 9 \\
$5-1$ & 5 & 1 \\
$5-5$ & 5 & 5 \\
$5-9$ & 5 & 9 \\
$9-1$ & 9 & 1 \\
$9-5$ & 9 & 5 \\
$9-9$ & 9 & 9 \\
\hline
\end{tabular}




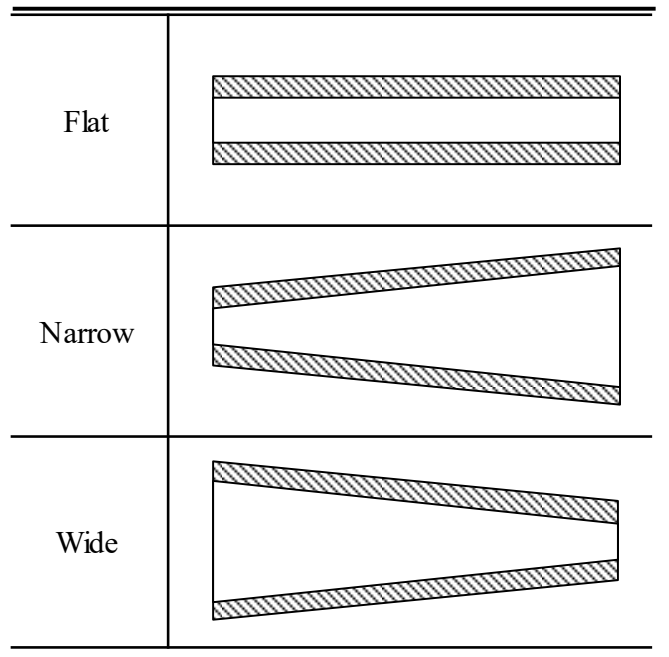

Figure 8. Types of slip shape at inlet.

\subsubsection{Initial Conditions}

Figure 9 shows the initial conditions for the numerical simulation. As shown, the human analog model was fixed to be perpendicular to the TSMD. One end of the tether extends from the TSMD section through the inlet and is fixed to the origin. The rigid system is arranged so that the tether and the TSMD section are aligned on the $X$ axis. We collected the tether and observed the motion of the rigid system that moves toward the origin while rotating. The measurement time of the motion was set to $10.0 \mathrm{~s}$ from the time when the tether ejection started. The fourth-order Runge-Kutta method was used for numerical integration. Table 2 shows the calculation parameters used in this study. These parameters are used based on the experimental equipment described in Reference [11]. In this parameter, mass of human analog is lighter than one of actual human, but there is no problem in investigating the contact mechanism.

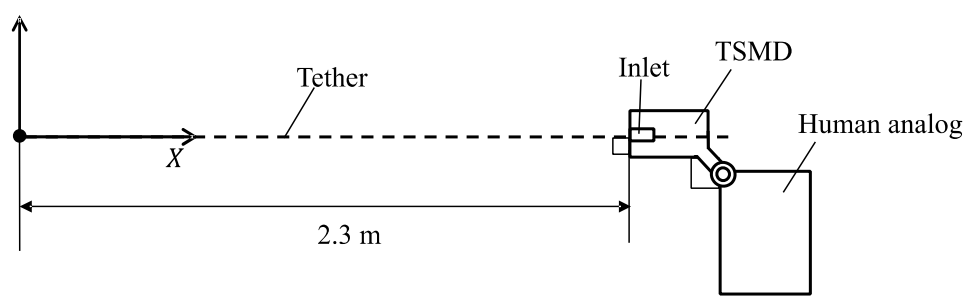

Figure 9. Diagram of initial conditions.

Table 2. Calculation parameters.

\begin{tabular}{cc}
\hline Parameter & Value \\
\hline Total length of tether, $l(\mathrm{~m})$ & 2.5 \\
Diameter of tether, $d(\mathrm{~m})$ & $5.2 \times 10^{-4}$ \\
Density of tether, $\rho\left(\mathrm{kg} / \mathrm{m}^{3}\right)$ & 1140 \\
Transverse elastic modulus, $E_{t}(\mathrm{GPa})$ & 1.93 \\
Longitudinal elastic modulus, $E_{l}(\mathrm{GPa})$ & 1.93 \\
Number of tether elements, $n$ & 50 \\
Mass of inlet, $M_{r 1}(\mathrm{~kg})$ & 0.0367 \\
Mass of TSMD part, $M_{r 2}(\mathrm{~kg})$ & 0.8172 \\
Mass of human analog, $M_{r 3}(\mathrm{~kg})$ & 7.78 \\
Moment of inertia of inlet, $I_{r 1}\left(\mathrm{~kg} \cdot \mathrm{m}^{2}\right)$ & $9.4 \times 10^{-6}$ \\
Moment of inertia of TSMD part, $I_{r 2}\left(\mathrm{~kg} \cdot \mathrm{m}^{2}\right)$ & $2.6 \times 10^{-3}$ \\
\hline
\end{tabular}


Table 2. Cont.

\begin{tabular}{cc}
\hline Parameter & Value \\
\hline Moment of inertia of human analog, $I_{r 3}\left(\mathrm{~kg} \cdot \mathrm{m}^{2}\right)$ & 0.0548 \\
Length of inlet, $l_{r 1}(\mathrm{~m})$ & 0.055 \\
Length of TSMD part $(\mathrm{m})$ & 0.17 \\
Length of human analog $(\mathrm{m})$ & 0.19 \\
Width of inner wall $(\mathrm{m})$ & 0.0015 \\
Width of TSMD part $(\mathrm{m})$ & 0.095 \\
Width of human analog $(\mathrm{m})$ & 0.22 \\
Control input $u_{+}$ & 0.01 \\
Control input $u_{-}$ & -0.06 \\
Spring constant of inner wall, $k_{1}(\mathrm{~N} / \mathrm{m})$ & 100 \\
Spring constant of edge, $k_{2}(\mathrm{~N} / \mathrm{m})$ & 100 \\
Damping coefficient of inner wall, $c_{1}(\mathrm{~N} /(\mathrm{m} / \mathrm{s}))$ & 0.0696 \\
Damping coefficient of edge, $c_{2}(\mathrm{~N} /(\mathrm{m} / \mathrm{s}))$ & 0.0696 \\
Coefficient of friction of inner wall of inlet, $\mu_{1}$ & 0.1 \\
Coefficient of friction of edge of inlet, $\mu_{2}$ & 0.1 \\
Coefficient of friction of inner wall of TSMD part & 0.0 \\
\hline
\end{tabular}

\subsection{Examination of Control Effect}

To examine the effect of the attitude control method (see Section 2.4), we compared the conditions for collecting the tether at a constant speed and the conditions for applying the control. Figure 10 shows the rotation angles of the rigid system for condition 1-1. As shown, the extension of the tether starts at $0.0 \mathrm{~s}$, and the winding starts when the tip of the tether sticks to the wall. For the rigid system, at 2.7 to $2.8 \mathrm{~s}$, the first tension acts and the rotation angle changes. Without control, after the second tension is applied to the tether at $4.0 \mathrm{~s}$, the tether is wound without tension and the rigid system continues to rotate at a constant angular velocity. With control applied, tension is applied to the tether at 5.0 and $7.0 \mathrm{~s}$, and thus the rigid system does not continue to rotate, and the rotation angle converges to a certain value. Similar results were obtained for other shapes, confirming that applying control suppressed the rotational motion of the rigid system under all conditions. Figure 11 shows the rotation angles of the rigid system for all shapes when control is applied. This figure shows that the time required for the rotation angle to converge to a constant value depends on the condition. The time required for the rotation angle to converge to a certain value followed the order condition 1-5, condition 1-1, and condition 1-9 (lowest to highest). This indicates that the control was responsive for a narrow inlet tip diameter, suggesting that there is a relationship between slit shape and control responsiveness. The effects of slit shape on tether tension and contact force are examined in detail in the next section.

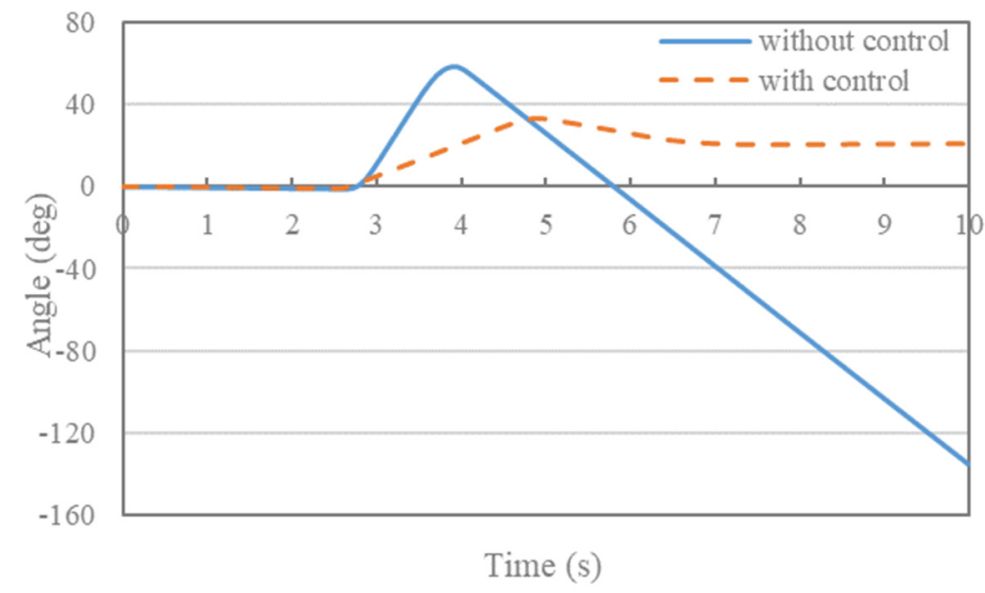

Figure 10. Time history of rotation angle for condition 1-1. 


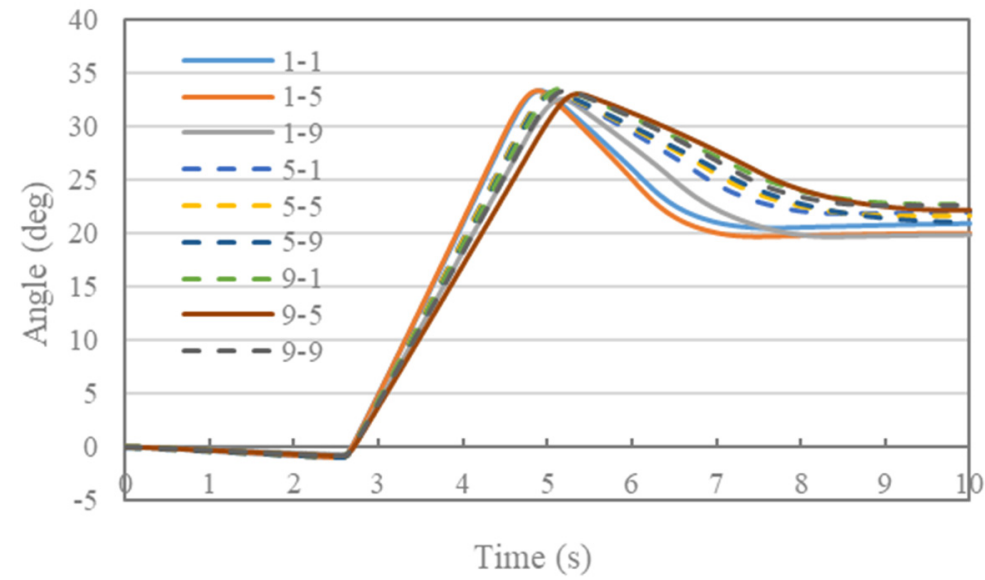

Figure 11. Time history of angle with control for various conditions.

\section{Mechanism of Effect of Slit Shape on Control Responsiveness}

The simulation results for the three conditions with an inlet tip diameter of $1 \mathrm{~mm}$, for which excellent control responsiveness was observed, are compared here. Figure 12 shows the time history of the control input for these conditions and Figure 13 shows the time history of the rotation angle of the rigid system, the tension acting on the tether, and the control input for condition 1-1. Figure 12 shows that the control input switched five times under the three conditions, and that a shorter convergence time for the rotation angle leads to earlier control input switching. Figure 13 confirms that the control input switches from negative to positive after a large tension is applied. The contact force inside the rigid body and that at the tip of the rigid body were compared in this section until the next control input was switched based on the time when the control input was switched under each condition.

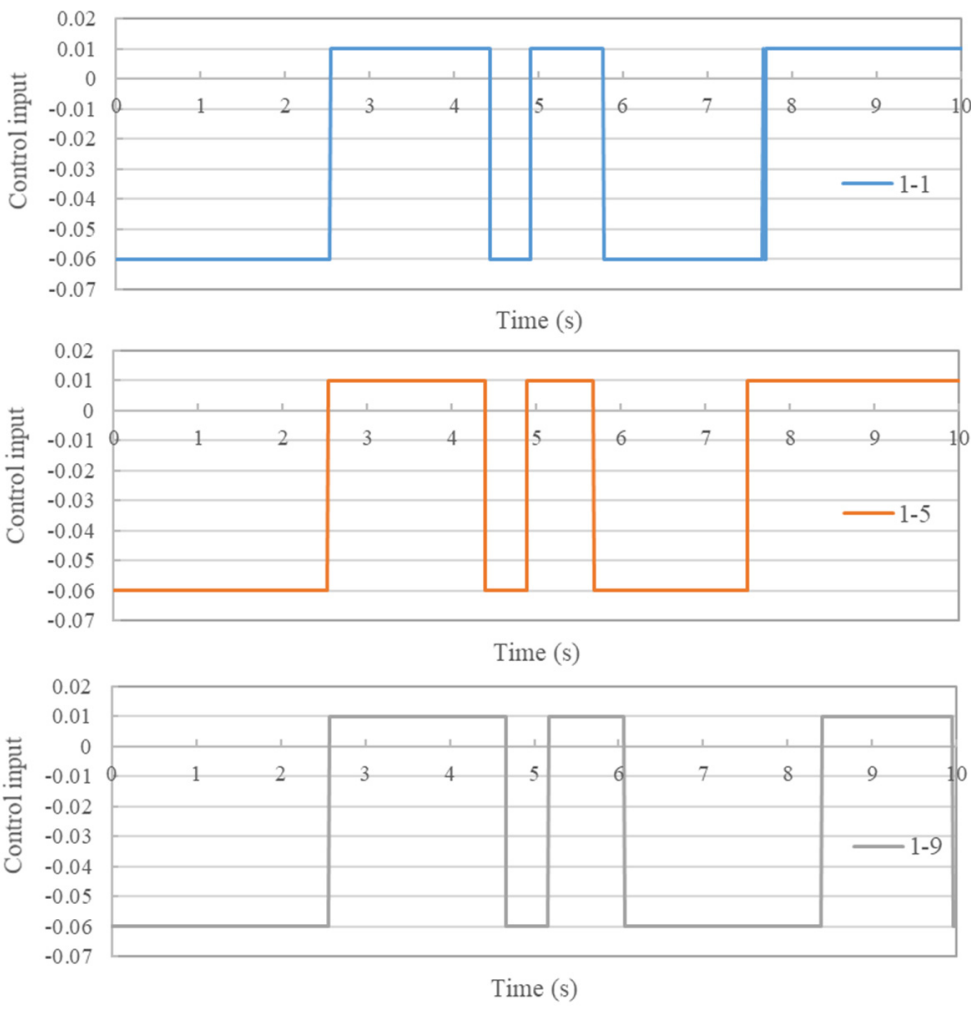

Figure 12. Time history of control input. 


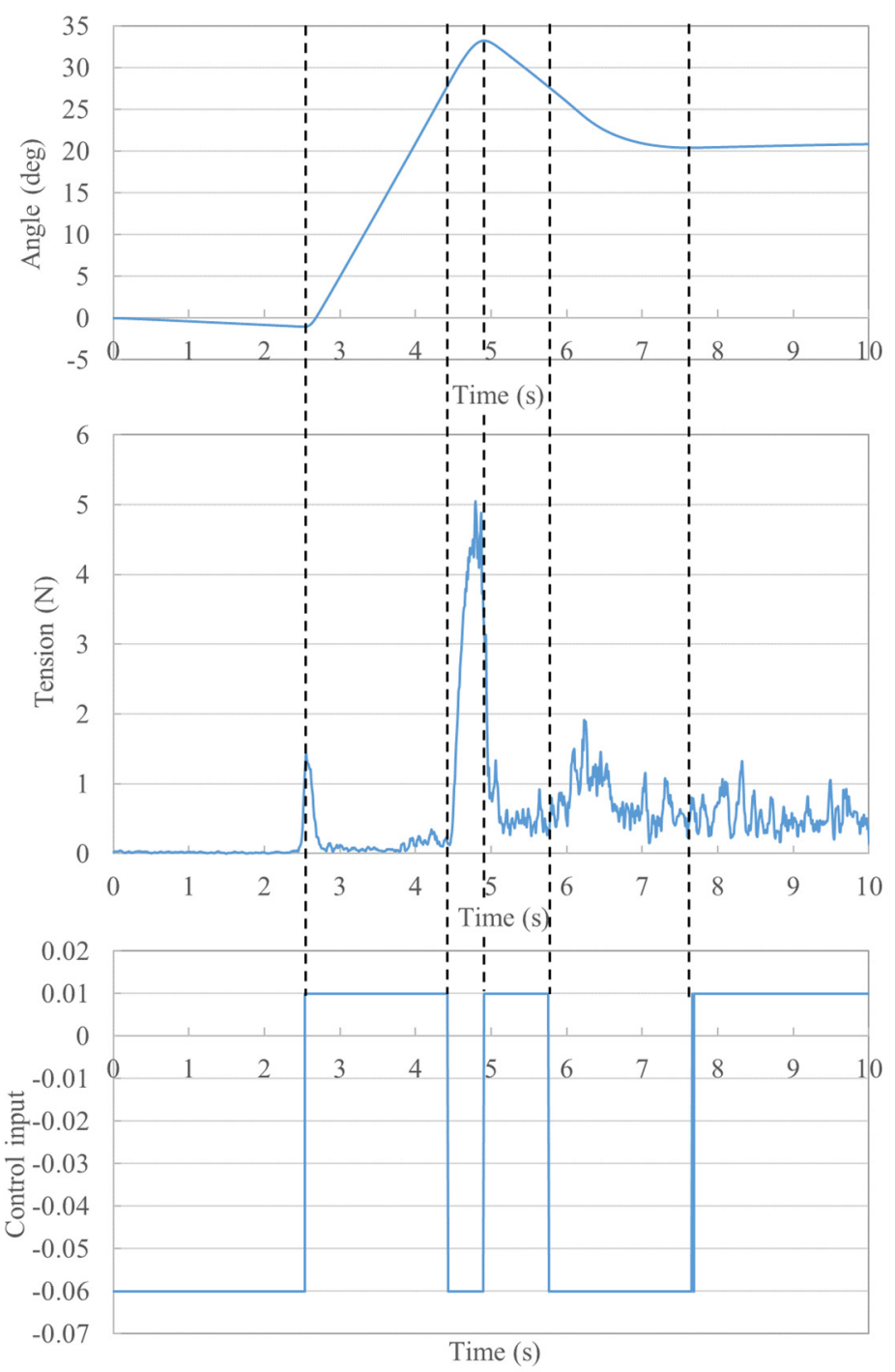

Figure 13. Time history of angle, tension, and control input for condition 1-1.

We compare the results from the start of tether extension until the tip of the tether stuck to the wall. Figure 14 shows the shape of the tether immediately after the tip of the tether stuck to the wall under each condition. For condition 1-9, the time required for the tip of the tether to stick to the wall was $0.04 \mathrm{~s}$ longer than that for other conditions. The range of angles at which one element of the tether can rotate inside the slit was the widest for this condition, causing the tether to bend more and thus it took more time for the tip of the tether to stick to the wall. This result indicates that if this range of angles is wide at the time of ejection, deflection is likely to occur, which would delay the switching of the control input. 


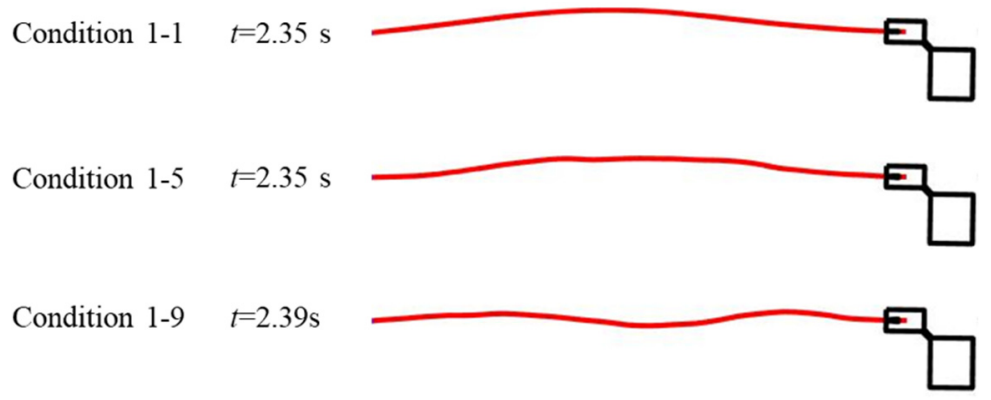

Figure 14. Shape of system during tether extension.

When the control input becomes positive at around $2.5 \mathrm{~s}$, the winding speed of the tether slows down. The results are compared in the section where the relative displacement between the inlet and the tether is small along the travel direction of the tether. In this section, the time until the next control input is switched is delayed by $0.03 \mathrm{~s}$ in condition 1-1 compared to that in condition 1-5. Here, because the angular velocity is always positive in this section, the control input changes depending on whether $(\varphi-\theta+\alpha)$ in Equation (16) is positive or negative. Figure 15 shows the time history of the contact force between the upper elastic wall and the tether inside the inlet (this force suppresses counterclockwise rotation). In this figure, the section from the first time when the control input becomes positive to the time when the control input becomes negative in Figure 12 is shown. The time when the control input is switched under each condition is $0.0 \mathrm{~s}$.

This figure shows that the contact force between the inlet and the tether is large when the slit width is narrow (e.g., condition 1-1). Based on the difference in contact force, it was confirmed that there was a delay in switching the control input under condition 1-1 compared to condition 1-5. At the time when the control input is switched and the tether winding speed decreases, the inlet and the tether are more likely to come into contact when the slit width is narrow (e.g., condition 1-1). The contact force delays control switching.

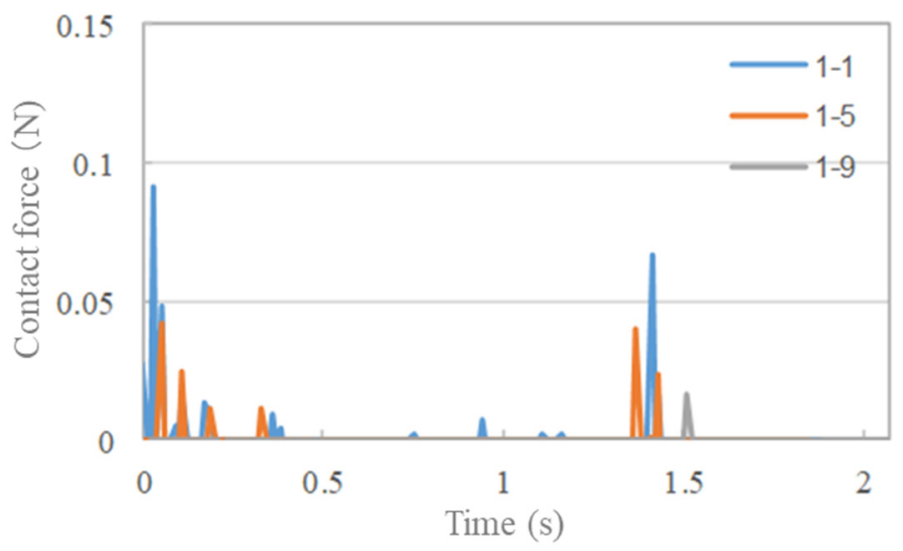

(a) Inner wall (upper side)

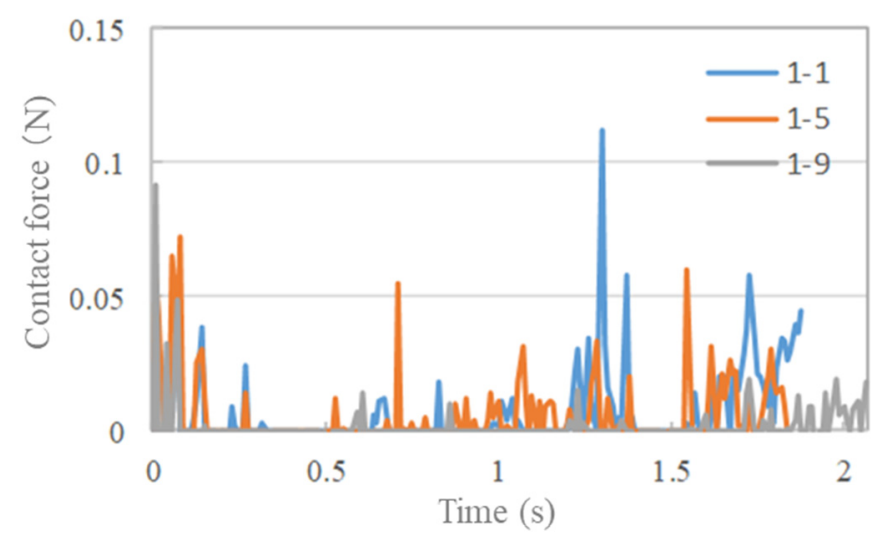

(b) Edge (upper side)

Figure 15. (a,b) Time history of contact force between upper elastic wall and tether inside inlet.

The winding speed greatly increased when the control input became negative. The results obtained until tension acts on the rigid body are compared. Figure 16 shows the time history of the moment due to the contact force between the tether and the rigid body when tension acts on the tether for various conditions and Figure 17 shows the distribution map of the contact force applied inside the rigid body immediately before the tension acts. Figure 17 shows the distance $X$ from the tip of the inlet to the contact position and the magnitude of the contact force in the longitudinal direction of the inlet. In Figure 16, the area under the graph can be considered to be the sum of the moments applied to the rigid body. This confirms that the moment due to the contact force between the tether and the 
rigid body increases in the order condition 1-5, condition 1-9, and condition 1-1. This order can be explained as follows. As shown in Figure 17, due to the inclination inside the inlet, a contact force acts on the tip side of the inlet many times under conditions 1-5 and 1-9. For a tapered inlet tip, even for a small taper angle, the contact is concentrated at the tip, improving control responsiveness. When the tip of the inlet is narrowed, and the second tension acts on the rigid system, the tip side of the inlet comes into more contact with the tether and the moment due to the contact force between the tether and the rigid body becomes large.

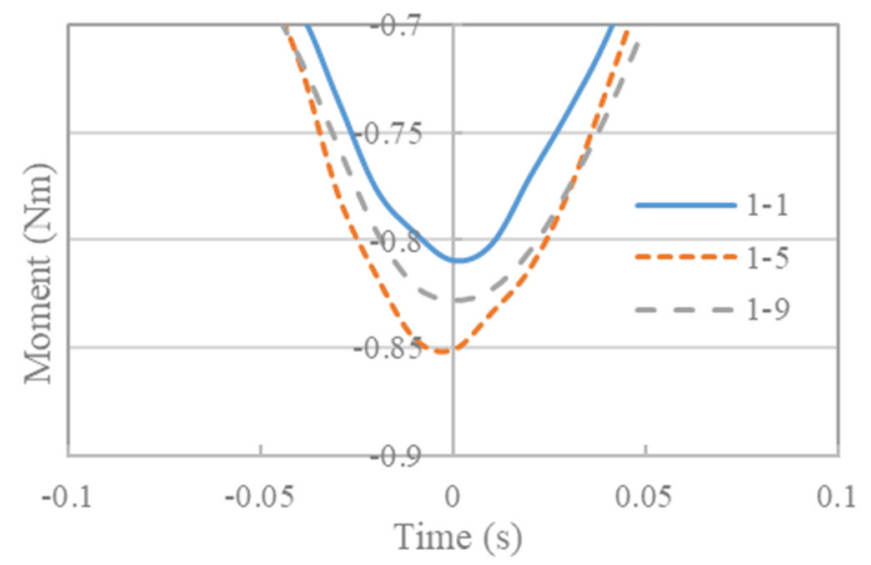

Figure 16. Time history of moment due to contact force.

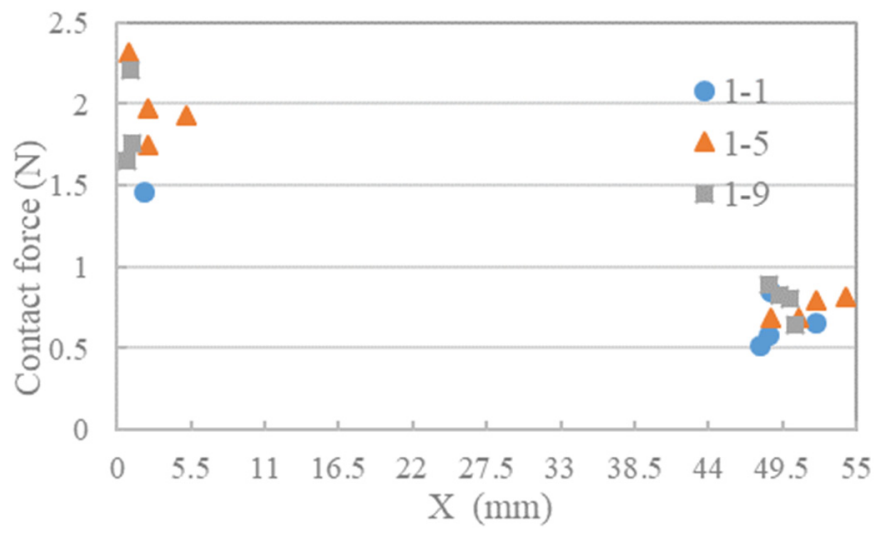

Figure 17. Contact force distribution.

Figure 18 shows the time history of the contact force between the inside of the inlet and the elastic wall at the tip on the upper side of the inlet at the time after the second tension was applied. For each condition, the difference in contact force is small at the time after the second tension is applied. Similar results were obtained for the lower side of the inlet. The control responsiveness was different due to the difference in the angle of the rigid body and the time when the control input was switched, which occurred before the second tension was applied. 


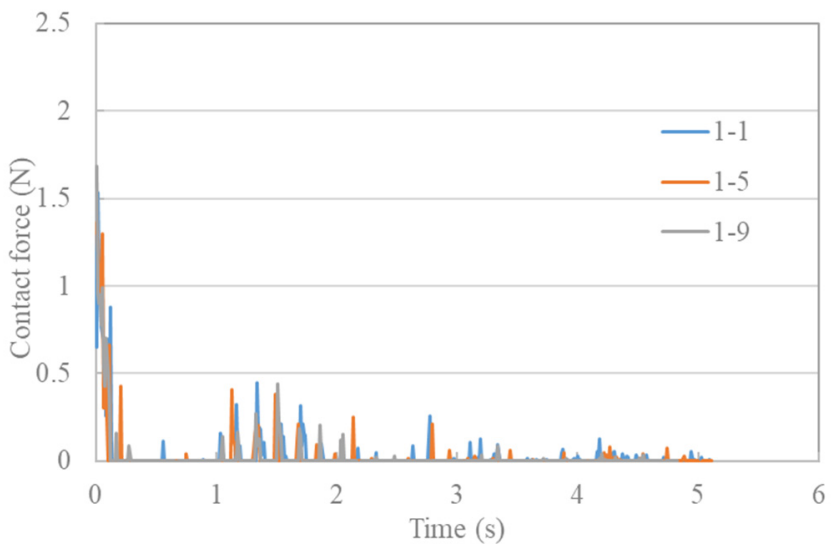

(a) Inner wall (upper side)

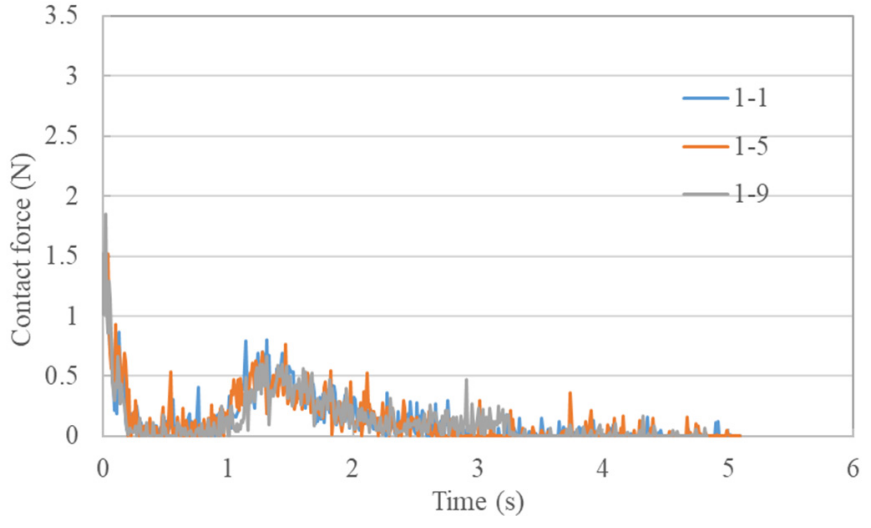

(b) Edge (upper side)

Figure 18. $(\mathbf{a}, \mathbf{b})$ Time history of contact force between inside of inlet and elastic wall at inlet tip after application of second tension.

The simulation results are compared in the same way for conditions with a large inlet tip diameter. Figure 19 shows the time history of the contact force inside the inlet and at the tip of the inlet on the upper side just before the second tension is applied. Here, condition 5-5 is used to represent the case with a uniform slit width and condition 9-1 is used to represent the case where the diameter at the tip of the inlet is much larger than that at the rear end of the inlet. Figure 19 confirms that a larger inlet tip diameter leads to a smaller contact force between the inlet tip and the inside. Similar results were obtained on the lower side of the inlet. Therefore, a larger inlet tip diameter leads to a larger relative distance between the elastic wall inside the rigid body and the tether and a smaller contact force; therefore, a moment is less likely to be applied. These results show that when the diameter of the inlet tip is large, the moment due to the contact force becomes small due to the large diameter of the inlet tip, the switching of the control input is delayed, and control responsiveness decreases.

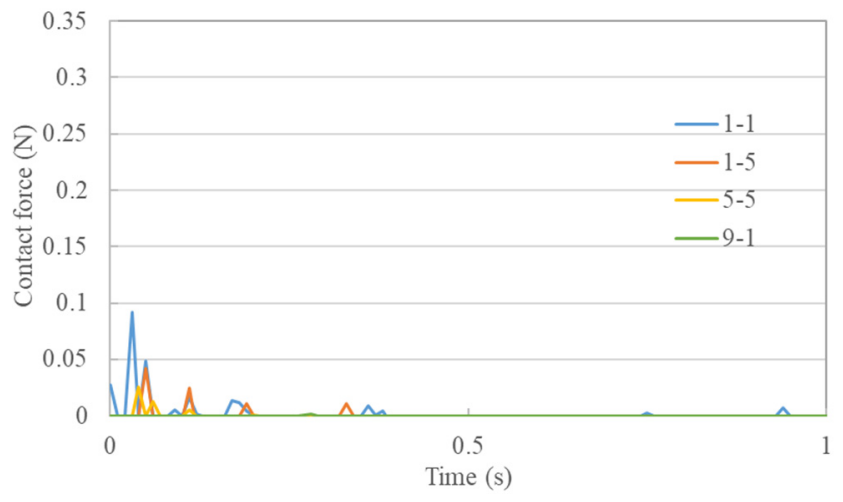

(a) Inner wall (upper side)

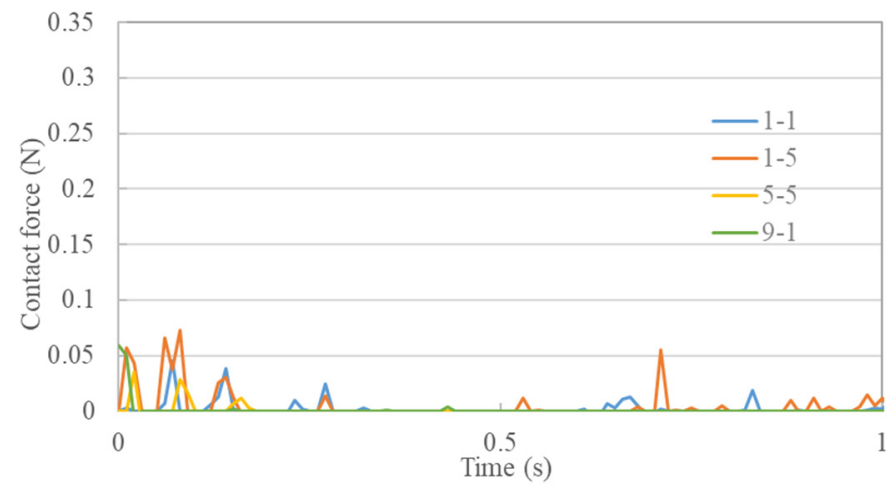

(b) Edge (upper side)

Figure 19. (a,b) Time history of contact force inside inlet and at tip of inlet just before application of second tension.

\section{Conclusions}

By observing the movement of the entire system during tether extension and winding, we investigated the effect of the contact force between the elastic wall and the tether at the slit of the elastic wall on the movement of the entire system. We created a model considering the slit shape to calculate the contact between the inlet and the tether. We could investigate the effect of slit shape during tether extension and winding on control responsiveness using numerical simulation due to the use of the detailed model. These results show when the tip of the inlet is narrowed, the tip side of the inlet comes into more contact with the tether and 
the moment due to the contact force between the tether and the rigid body becomes large. In addition, when the diameter of the inlet tip is large, the moment due to the contact force becomes small due to the large diameter of the inlet tip, the switching of the control input is delayed, and control responsiveness decreases. These results show that property tapering the slit shape of the inlet improves control responsiveness. In addition, we obtained the above results by performing detailed modeling of slits. Thus, it is showed that the detailed model is useful to design the machine including control system.

Author Contributions: S.T. (Satoshi Takayama), S.T. (Shoichiro Takehara) and R.Y. formulated the numerical model; R.Y. coded the numerical model; S.T. (Satoshi Takayama), S.T. (Shoichiro Takehara) and R.Y. analyzed the data and discussed the results; S.T. (Satoshi Takayama) wrote the paper. All authors have read and agreed to the published version of the manuscript.

Funding: This research was funded by JSPS KAKENHI grant number [JP26820075].

Institutional Review Board Statement: Not applicable.

Informed Consent Statement: Not applicable.

Data Availability Statement: Not applicable.

Conflicts of Interest: The authors declare no conflict of interest.

\section{References}

1. Modi, V.J; Misara, A.K. On the deployment of tether connected two-body systems. Acta Astronutica 1979, 6, 1183-1197. [CrossRef]

2. Nohmi, M. Space verification experimental analysis for attitude control of a tethered space robot. Trans. JSME 2014, 80, SE0282.

3. Takehara, S.; Nishizawa, T.; Kawarada, M.; Hase, K.; Terumichi, Y. Development of Tether Space Mobility Device. Comput. Methods Appl. Sci. 2014, 35, 255-274.

4. Carrier, G.F. The Spaghetti Problem. Am. Math. Mon. 1949, 56, 669-672. [CrossRef]

5. Shabana, A.A. Dynamics of Multibody Systems, 3rd ed.; Cambridge University Press: Cambridge, UK, 2005.

6. Wago, T.; Kobayashi, N.; Sugawara, Y. Improvement on evaluating axial elastic force in beam element based on the absolute nodal coordinate formulation by accurate mean axial strain measure. Trans. Jpn. Soc. Mech. Eng. 2013, 79, 2704-2713. (In Japanese) [CrossRef]

7. Berzeri, M.; Shabana, A.A. Development of simple models for the elastic forces in the absolute nodal co-ordinate formulation. J. Sound Vib. 2000, 235, 539-565. [CrossRef]

8. Shabana, A.A. Dynamics of Multibody Systems, 2nd ed.; Cambridge University Press: Cambridge, UK, 1998.

9. Takehara, S.; Kawarada, M.; Hase, K. Dynamic Contact between a Wire Rope and a Pulley Using Absolute Nodal Coordinate Formulation. Machines 2016, 4, 4. [CrossRef]

10. Kobayashi, N.; Komaki, Y.; Watanabe, M. Effect of gap size at inlet on spaghetti problem. Trans. Jpn. Soc. Mech. Eng. 2001, 67, 641-647. (In Japanese) [CrossRef]

11. Takehara, S.; Uematsu, Y.; Miyaji, W. Tether Space Mobility Device Attitude Control during Tether Extension and Winding. Machines 2018, 6, 4. [CrossRef]

12. Sato, S.; Tao, K.; Nohmi, M.; Yamagiwa, Y.; Aoki, Y.; Ootsuka, K.; Ishikawa, Y. Analysis of tether deployment dynamics of tether microsatellite system. In Proceedings of the Space Transportation Symposium FY2016, Sagamihara, Japan, $19-20$ January 2017; pp. 1-6. 